\title{
Quantitative Mass Spectrometry Imaging of Molecules in Biological Systems
}

Ingela Lanekoff ${ }^{1}$ and Julia Laskin ${ }^{2 *}$

1) Dept. of Chemistry - BMC, Uppsala University, Box 599, 75124 Uppsala, Sweden

2) Physical Sciences Division, Pacific Northwest National Laboratory, P.O. Box 999, MSIN K8-88, Richland, WA 99352, USA

E-mail: Ingela.Lanekoff@kemi.uu.se,Julia.Laskin@pnnl.gov

*Authors for correspondence

Keywords: Mass spectrometry imaging, MSI, matrix effects, quantification, quantitation, Q-MSI 


\section{Abstract}

Mass spectrometry imaging (MSI) enables label-free, spatially_-resolved analysis of molecules in biological samples. MSI coupled with soft ionization techniques provides two- and threedimensional maps of intact molecular ions, which greatly simplifies mass spectra and facilitates identification of molecules extracted from the sample. However, differences in ionization efficiencies of biomolecules and signal suppression during ionization, also referred to as "matrix effects," represent a challenge for quantitative MSI (Q-MSI) experiments. In this review, we summarize the current understanding of matrix effects in MSI and discuss different quantification strategies. We emphasize that using internal standards in Q-MSI is essential for accurate imaging and quantification of molecules in chemically complex samples. The ability to localize, identify, and quantify molecules in tissue sections and cells opens up unique opportunities for molecular-level understanding of biological processes, elucidation of disease mechanisms, understanding drug interactions with living organisms, and controlling synthesis of natural products. These capabilities make Q-MSI a powerful tool for biological research, clinical studies, and drug development.

\section{Introduction to mass spectrometry imaging}

Mass spectrometry imaging (MSI) has been extensively used for obtaining spatial distributions of endogenous molecules (e.g., lipids, metabolites, and proteins), as well as drugs and their metabolites in biological samples. When combined with soft ionization techniques, MSI generates two- and three-dimensional maps of intact molecules extracted from specific locations on the sample. In a typical MSI experiment, a mass spectrum containing hundreds of features corresponding to analyte molecules extracted from the sample is obtained in each pixel. Ion images are generated by plotting ion intensities in individual mass spectra as a function of location on the sample. Because signal intensities in mass spectra are affected by several factors, including ionization efficiency of the analyte molecules, analyte concentration, and sample composition, quantification in MSI experiments is challenging. In particular, matrix effects defined as signal suppression during direct ionization of complex mixtures may affect the observed ion images and complicate quantification. Nevertheless, several strategies have been developed for both relative and absolute quantification in MSI. This review summarizes the current status of quantitative MSI (Q-MSI) and discusses future directions in this exciting field. The discussion will focus on several soft ionization techniques, where substantial effort has been dedicated to developing Q-MSI approaches.

By far, matrix-assisted laser desorption ionization (MALDI) is the most broadly used soft ionization technique in MSI experiments. MALDI imaging sources are commercially available and have been coupled to several mass analyzers. Time-of-flight (TOF) analyzers are particularly attractive because of their relatively low cost, good mass accuracy, fast data acquisition, and almost unlimited mass range. Both low- $(m / \Delta m<5,000)$ and high-resolution $(\mathrm{m} / \Delta m \sim 40,000)$ TOF instruments have been used in MALDI-MSI experiments. Meanwhile, high-resolution Orbitrap and Fourier transform ion cyclotron resonance (FT-ICR) instruments provide the advantages of excellent mass separation $(m / \Delta m>60,000)$ at the expense of acquisition rate. High-resolution mass analysis is important for separating isobaric species commonly observed in MSI of chemically complex biological samples. Meanwhile, high mass accuracy facilitates peaks assignments in mass spectra. MALDI-MSI experiments have been performed both in vacuum and under ambient conditions with a typical spatial resolution of $\sim 100 \mu \mathrm{m}$. Although somewhat less sensitive than vacuum MALDI, atmospheric 
pressure (AP)-MALDI eliminates the need of sample introduction into a vacuum system and is more readily adapted to different mass spectrometers.[1] AP-MALDI with a spatial resolution of better than $5 \mu \mathrm{m}$ has been used to generate histology-like molecular images of tissue sections.[2] Similarly, AP-MALDI combined with electrospray ionization (MALDESI) has been used for imaging biological samples under ambient conditions.[3]

Despite the wide use of MALDI-MSI, the requirement of matrix application prior to analysis, which is undesirable for some applications, has prompted development of ambient ionization techniques.[4-7] Ambient ionization enables analysis of biological samples without special sample pretreatment, which simplifies sample preparation protocols and eliminates the possible effect of matrix application on the localization of molecules in the sample.[4-6, 8-11] A broad range of ambient ionization techniques developed in the past decade can be classified into methods that rely either on liquid extraction or involve more energetic desorption of analytes from the sample using laser or plasma bombardment.[12] In this review, we will limit our discussion to liquid-extractionbased ambient ionization techniques.[13]

Desorption electrospray ionization (DESI) is one of the most popular liquid extraction ambient ionization techniques that has been successfully coupled with MSI. In DESI, the sample is bombarded with charged droplets accelerated toward the sample surface via a sheath gas flow. This process generates secondary charged droplets that contain analyte molecules extracted from a specific location on the sample. Subsequent desolvation of the secondary droplets generates ions corresponding to intact molecules present in the sample. Several variants of DESI have been developed to improve this technique's sensitivity to specific classes of analytes and its spatial resolution. The best spatial resolution of $35 \mu \mathrm{m}$ has been reported in DESI-MSI of mouse brain tissue sections.[14]

Another family of ambient ionization techniques relies on direct extraction of molecules into a liquid bridge formed between a specially designed sampling probe and the sample surface.[13] Different configurations of direct liquid extraction probes have been summarized in a recent review and, hence, will not be discussed here.[13] Instead, we will focus on two direct liquid extraction ambient ionization techniques that have been used in Q-MSI experiments. Specifically, we will limit our discussion to liquid extraction surface analysis (LESA)[15] and nanospray desorption electrospray ionization (nano-DESI)[16] mass spectrometry. In LESA, analyte is first extracted into a static small droplet placed on the sample surface and subsequently placed in front of a mass spectrometer for nanoelectrospray ionization. In contrast, in nano-DESI, the analyte is extracted into a flowing solvent delivered to the sample by a primary fused silica capillary and removed through a secondary capillary that transfers the extracted analyte to a mass spectrometer inlet. The two capillaries comprise the nano-DESI probe. The difference in the respective probe designs translates directly into differences in spatial resolution. Specifically, the typical spatial resolution of LESA is $\sim 1 \mathrm{~mm}[17]$, while the best spatial resolution of $\sim 350 \mu \mathrm{m}$ has been achieved using an electrofocusing LESA probe.[18] In contrast, the typical spatial resolution of nano-DESI MSI is $\sim 100-150 \mu \mathrm{m}[19-22]$, while the best reported value is $\sim 12 \mu \mathrm{m}$.[23]

Quantification strategies discussed in this review rely on using standards of known concentration. In Q-MSI experiments, one or more standards are either deposited directly onto the sample or delivered to the sample via the extraction solvent. In the following sections, we will 
provide a detailed summary of these approaches and highlight some of the key applications of Q-MSI in biological research. Q-MSI is a rapidly growing field poised to transform MSI into an analytical technique that will enable accurate measurement of chemical gradients of hundreds of molecules in biological samples.

\section{Matrix effects in MSI}

Q-MSI experiments rely on the assumption that signal intensities in mass spectra obtained in individual pixels are proportional to analyte concentrations at each location. We begin by discussing "matrix effects" that may challenge this assumption and affect ion images obtained in MSI experiments. Matrix effects, also referred to as ion suppression effects, occur during ionization of analyte mixtures as a result of molecules competing for charge.[24-29] Matrix effects occur in all ionization techniques and, as such, are not unique to MSI. They also affect ionization in liquid chromatography mass spectrometry (LC-MS) experiments.[30] However, due to the nature of MSI, where all molecules from a specific location on the sample surface are ionized simultaneously, matrix effects originating from the sampled microenvironment may be more severe than in LC-MS. Matrix effects typically are studied and compensated for using an internal standard. Different approaches for applying internal standards will be discussed in more detail in the sections herein describing quantification. In the following sections, we will discuss two types of matrix effects that are particularly important in MSI: 1) ionization suppression due to the analyte mixture's molecular composition and 2) variation in ionization efficiency resulting from differences in the alkali metal concentrations in the sample.

\section{Matrix effects due to the molecular composition}

During competitive ionization, molecules having a high affinity for charge suppress ionization of molecules with lower affinity for charge. Therefore, the analyte mixture's molecular composition may significantly influence signal intensities obtained for individual compounds. As a result, signal intensities may not accurately reflect the concentration on the analyte molecules in the sample. The presence of matrix effects in MSI has been reported for different ionization techniques and various sample types.[26-28, 31-52] The primary conclusion of these reports is that it is possible to compensate for matrix effects during MSI by incorporating a carefully selected internal standard. To compensate for matrix effects, the internal standard must have the same ionization efficiency as the target analyte. For example, Prideaux et al. used the internal standard levofloxacin homogenously sprayed over the tissue section to accurately determine the distribution of the drug moxifloxacin in rabbit lung tissue using MALDI-MSI.[44] The resulting ion image of the internal standard levofloxacin, shown in Figure 1B, displays distinct regions of higher intensity, indicating differences in matrix effects across the lung tissue section. Figure $1 \mathrm{C}$ shows the ion image of the administered drug, moxifloxacin, affected by matrix effects. By normalizing the intensity of moxifloxacin to the intensity of the internal standard levofloxacin, an ion image free of matrix effects is generated. This ion image, shown in Figure 1D, provides an accurate representation of the distribution of moxifloxacin in rabbit lung.[44] In another study, the distribution of levofloxacin in lung tissue post administration was found to be similar to the distribution of moxifloxacin. This result was obtained using deuterated levofloxacin as an internal standard added to the MALDI matrix solution.[47] In a related study, Pirman et al. used an internal standard to study organ-specific matrix effects in MALDI-MSI of acetylcarnitine [53] and found that different chemical compositions extracted from various organs 
had a pronounced impact on the internal standard's observed intensity.[53] Similarly, Rosen et al. examined tissue-specific matrix effects by imaging a thin tissue section of neonatal rat using infrared (IR)-MALDESI.[39]

Dong et al. investigated different strategies for introducing internal standards into DESI-MSI experiments.[32] They used glutaric acid as an internal standard to visualize the distribution of structurally similar organic acids in grapevine stem. Figure 2A shows ion images obtained for a stem that was soaked in the internal standard prior to DESI analysis, while ion images shown in Figure $2 \mathrm{~B}$ were obtained by adding the internal standard to the DESI solvent during analysis. The top and middle rows of both Figures $2 \mathrm{~A}$ and $2 \mathrm{~B}$ show ion images of the internal standard and the analytes normalized to the total ion current (TIC) that include matrix effects. The third row shows ion images of the analytes normalized to the internal standard, which compensates for matrix effects. The normalized ion images shown in Figures $2 \mathrm{~A}$ and $2 \mathrm{~B}$ differ strikingly, indicating that the approach used for incorporating the internal standard may affect the observed ion distributions. The authors suggest that if validation is not performed, differences in specific physicochemical interactions over the tissue surface may skew the results and cause misinterpretation of the obtained data.[32]

Matrix effects also have been investigated using nano-DESI.[33, 36, 42, 54] In these studies, an internal standard is added to the nano-DESI solvent and supplied at a constant rate throughout the imaging experiment. Non-uniform distributions of nicotine-d used as a standard were observed in nano-DESI MSI of rat brain tissue sections, indicating the presence of matrix effects. Prior to normalization to the internal standard, nicotine was localized in the white matter regions, while normalization to the nicotine-d signal revealed a significantly different image free of matrix effects. This image revealed enhanced abundance of nicotine in the cortex and dentate gyrus.[33] Bergman et al. used nano-DESI MSI to investigate the impact of matrix effects on the distribution of small endogenous neurotransmitters in thin rat brain tissue sections by adding deuterated internal standards to the nano-DESI solvent.[36] Figures 3a-c show ion images of the deuterated standards, acetylcholine (ACh-D9), $\gamma$-aminobutyric acid (GABA-D2), and glutamate (Glu-D3). Despite the uniform supply of the internal standards, the ion images show regions of increased intensities, suggesting reduced matrix effects in these regions. Figures $3 \mathrm{~d}-\mathrm{f}$ show the distribution of endogenous acetylcholine (ACh), $\gamma$-aminobutyric acid (GABA), and glutamate (Glu). These ion images include any matrix effects. The ion images displayed in the third row, Figures $3 g-i$ are normalized to the intensity of the respective internal standard in every pixel. Free of matrix effects, these ion images more accurately represent the spatial localization of neurotransmitters in rat brain tissue.[36] Lanekoff et al. examined matrix effects involved during imaging of phospholipids using nano-DESI MSI.[43] In these experiments, two non-natural phosphatidylcholines (PC) were used as internal standards. The native distribution of several PC species was determined by normalizing ion signals observed for these endogenous species to the signal intensity of the appropriate adduct of one of the internal standards. They found that the acyl chain length of individual PC species had an effect on the observed ion suppression. Specifically, lower ion signals were obtained for PC species containing short acyl chains, indicating more substantial suppression of these species in comparison to PC containing longer acyl chains during ionization of a complex chemical mixture extracted from the tissue sample.[54] 


\section{Matrix effects due to cation abundances}

Although matrix effects resulting from variations in alkali metal concentrations in biological samples have received less attention, the presence of such ion suppression effects has been discussed in the literature. By nature, the distribution of alkali metal cations in biological tissues may be fairly heterogeneous. Furthermore, tissue cryo-sectioning prior to MSI may introduce additional heterogeneity that originates from the plane through which the cells are sectioned.[55] Crosssectioned cells typically display high amounts of potassium, while intact cells exhibit higher levels of sodium contained in the outer plasma membrane.[55] Because of the relatively high concentration of sodium and potassium in tissue sections, $[\mathrm{M}+\mathrm{Na}]^{+}$and $[\mathrm{M}+\mathrm{K}]^{+}$ions often dominate mass spectra obtained in $\mathrm{MSI}$ experiments. Of note, the preference for protonation, cationization on sodium, and cationization on potassium varies between molecular classes, which determines the relative abundance of different adducts produced during ionization. Naturally occurring variations in cation abundances on the sample surface may influence the observed signal intensities of different adducts in MSI. Recent advancements in MSI instrumentation enable imaging experiments with high spatial resolution approaching subcellular level (greater than 10 microns).[2] Under these conditions, the sample surface's local microenvironment may increase pixel-to-pixel variations in the ion image because of the differences in matrix effects. For example, a spectrum acquired from a larger area $\left(\sim 100 \times 100 \mu^{2}\right)$ is an average of approximately 25 cells, and the plane through which individual cells have been sectioned may not have any substantial impact on the observed signal. However, imaging with higher spatial resolution of about $10 \mu \mathrm{m}$ enables sampling of individual cells in each pixel. This may result in substantial scan-to-scan variations due to the increased heterogeneity of the chemical composition examined in each scan. Therefore, it is particularly important to account for matrix effects in MSI experiments performed with high spatial resolution.

To minimize the influence of matrix effects resulting from variations in cation abundances in tissue sections, Cerruti et al. added lithium to the uniformly applied matrix solution prior to MALDIMSI.[56] When lithium concentration greatly exceeds the concentration of other cations in the sample, all molecules are preferentially cationized on lithium. They demonstrated that the exclusive cationization on lithium reduces matrix effects resulting from local variations of cation concentrations in the sample.[56] A second reported strategy to compensate for varying cation concentrations over the sample surface involves desalting tissue sections prior to MALDI-MSI.[57-59] Desalting is performed by drip-washing the sample in ammonium acetate followed by vacuum drying and matrix application.[57] To eliminate the effect of increased sodium and decreased potassium concentration in the ischemic region on results obtained in MALDI-MSI experiments, Wang et al. used desalted brain tissue sections of ischemic stroke models.[57] Lanekoff et al. developed a strategy that allows compensating for matrix effects in nano-DESI MSI resulting from heterogeneity in cation abundances by adding internal standards to the nano-DESI solvent and normalizing ion signals obtained for endogenous phospholipids to those of the corresponding standard's alkali metal adducts.[42] Figure 4 shows four endogenous PC species and their distributions in brain tissue sections of a mouse model of ischemic stroke. Figures $4 A$ and B depict ion images of sodium, $[\mathrm{M}+\mathrm{Na}]^{+}$, and potassium adducts, $[\mathrm{M}+\mathrm{K}]^{+}$, of these $\mathrm{PC}$ species, respectively. These ion images show an enhanced abundance of $[\mathrm{M}+\mathrm{Na}]^{+}$and reduced abundance of $[\mathrm{M}+\mathrm{K}]^{+}$ions in the ischemic region, which is attributed to matrix effects. Figures $4 C$ and D show the same ion images after normalization to the internal standard, PC 25:0 (total number of carbons:total number of double bonds), using the appropriate alkali cation adduct. Similar ion images obtained for $[\mathrm{M}+\mathrm{Na}]^{+}$and $[\mathrm{M}+\mathrm{K}]^{+}$species after 
normalization demonstrate efficient compensation for matrix effects, which helps reveal the true distribution of various PC species in the sample.[42]

\section{Q-MSI}

Compensation for matrix effects is an important prerequisite for Q-MSI experiments that enable simultaneous analysis of the amount and localization of compounds in biological samples, including thin tissue sections. In addition, an accurate determination of analyte desorption efficiency from different locations on the sample is required for converting the observed signal intensity into both relative and absolute amounts of the analyte present in the sample. Both matrix effects and desorption efficiencies are influenced by the physical properties and chemical composition of the sample and must be taken into account in every pixel for each analyte ion. An additional challenge, specific to MALDI-MSI, is to ensure high quality and uniformity of the applied MALDI matrix resulting in formation of small crystals on the sample surface. For the interested reader, specific challenges for MALDI-MSI have been extensively discussed in several recent reviews.[26, 27, 29, 38, 46, 50, 60-63]

LC-MS or LC-MS/MS experiments often are used to validate Q-MSI results.[31, 44, 47, 48, 55, 64-75] LC-MS/MS is preferentially used in quantitative studies to ensure that isobaric species do not interfere with quantification. For the same reason, MSI uses MS/MS and/or mass spectrometers with high mass resolving power for quantitative analyses. When combining MS/MS-based quantification and an isotopically labeled standard, a wide isolation window, which enables simultaneous detection of fragment ions of both the analyte and standard in the same scan, typically is used.[31, 38, 65] Reyzer et al. quantified drug candidates in brain tissue using LC-MS/MS and compared the results with the intensities acquired using MALDI-MSI.[65] They concluded that the intensities obtained by MALDI-MSI reflected the relative amount of the drug in tissue as determined by LC-MS/MS. However, they proposed that because of the chemical heterogeneity of brain tissue, the intensities obtained from a single thin tissue section may not correlate with results obtained from a homogenate of the whole brain analyzed by LC-MS/MS.[65] To compensate for the chemical heterogeneity of brain tissue, Hankin et al. performed quantitative LC-MS/MS analysis of PC species in selected regions of rat brain tissue extracted using microdissection.[55] The intensities of four abundant PC species acquired using MALDI-MSI then were compared with the results of LC-MS/MS experiments. After the data were normalized to the intensity of the PC 34:1, a reasonable agreement between MALDI-MSI and LC-MS/MS was obtained.[55] Chumbley et al. investigated how different applications of an internal standard impacted the quantitative correlation of MALDI-MSI to LCMS/MS. The best correlation of Q-MSI and quantitative LC-MS/MS was obtained when matrix application was performed after applying standards onto the tissue section.[31] Several other techniques also have been used for developing and validating Q-MSI. For example, gas chromatography (GC),[50] Raman spectroscopy imaging,[76] surface plasmon resonance imaging,[77] magnetic resonance imaging,[78] positron emission tomography,[79] and autoradiography[80-82] have been correlated with MSI. The term "Q-MSI" is interchangeably used to describe experiments involving either relative or absolute quantification (described in more detail in the following sections).

\section{Relative quantification with MSI}

Relative Q-MSI typically refers to an experiment, where the absolute ion intensity of a compound of interest in each pixel of the ion image is converted into the relative abundance of the 
compound. This approach facilitates comparison between different samples or regions in the sample.[83] Relative Q-MSI is achieved either by defining compound-specific response factors (discussed in more detail later in this review) $[45,51,72,84]$ or by introducing internal standards [32, $35,36,69,72,75,85,86]$ to compensate for matrix effects.[60] Both strategies have been applied successfully for relative quantification. Using MALDI-MSI, Stoeckli et al. performed relative quantification by determining response factors for a drug candidate in different organs of a whole body tissue section.[45] Subsequently, organ-specific response factors were used for relative quantification of the drug candidate in different organs after in vivo administration (Figure 5).[45] A second strategy for relative quantification is to normalize the analyte signal intensity to that of deuterated standards. For relative quantification of cocaine and cocaine metabolites in hair strands, Porta et al. used deuterated internal standards sprayed onto the sample prior to MALDI-MSI.[72] The relative concentration of cocaine and its metabolites at different distances from the scalp were determined using the ratio of the ion intensity observed for each compound to the intensity of the respective deuterated standard.[72] Similarly, Schultz et al. reported relative quantification of the drug dasatinib in kidney tissue sections of differently treated animals using MALDI-MSI and a homologous distributed deuterated internal standard.[69] The intensity ratio of the ion signals obtained for dasatinib to the deuterated analog in one region of the tissue section was divided by the intensity ratio of the drug's total signal in the whole tissue section to the total signal of the deuterated analog in the whole tissue section. This approach eliminated differences in matrix effects and desorption efficiencies between different tissue sections, thereby enabling relative quantification.[69]

\section{Absolute quantification with MSI}

Experiments in which signal intensities in individual pixels of the ion image are converted into absolute abundances of specific analyte molecules reported in grams or moles per gram tissue are typically referred to as "absolute Q-MSI." In absolute Q-MSI, quantification is performed using an external calibration curve.[60, 83] The analysis generally is performed by spotting calibration standards at different concentrations onto control tissue sections followed by MSI to generate an external standard calibration curve. The curve subsequently is used to convert the acquired signal intensity into analyte concentration at a particular location.[44, 49, 50, 53, 61, 66, 70, 71, 79, 87, 88] The weight of a single tissue section then is used to extrapolate the obtained concentrations to amount per tissue weight. However, because of the natural chemical heterogeneity of tissue samples, this approach does not necessarily account for matrix effects during MSI.[31] Källbeck et al. investigated how external standard curves, obtained after spotting calibration standards on a lung tissue section, were affected by using different normalization strategies.[49] Figure 6 shows the calibration curves obtained by engaging different normalization approaches evaluated in that study. Improved quantification was obtained using a deuterated internal standard, uniformly deposited onto the tissue section. This improvement was attributed to more efficient compensation for matrix effects originating from the tissue's molecular composition and suggests this was the best strategy for accurate quantification.[49] Lagarrigue et al. performed absolute quantification of a pesticide in mouse liver using an isotopically labeled standard and correlated the results to GC-electron capture detector (ECD).[50] Bokhart et al. performed absolute quantification of the drug emtricitabine in tissue using IR-MALDESI by normalizing the analyte signal to that of the internal standard.[71] The 
following sections will describe several successful strategies for both on- and off-line quantification using internal standards.

\section{Approaches for Q-MSI}

Approaches for Q-MSI involve using one or more internal standards, usually isotopically labeled analogs or unlabeled homologs of the analyte to be quantified. An isotopically labeled internal standard has the same physiochemical properties and ionization efficiency as the analyte and, therefore, experiences the same matrix effects as the extracted analyte molecules. Similarly, homologous compounds have similar ionization efficiency as analytes of interest and are assumed to experience comparable suppression during ionization. Several approaches have been developed for introducing the internal standard into Q-MSI, including offline strategies, where the internal standard intensity is measured separately from the analyte, and online strategies, where the internal standard intensity is measured simultaneously with the analyte.

\section{Offline strategies}

Offline strategies can be divided into the following three categories (shown schematically in Figure 7): A) applying the internal standard onto a separate tissue section to obtain a calibration curve, B) applying the internal standard onto a separate tissue section to determine response factors, and $\mathrm{C}$ ) incorporating the internal standard into a homogenized tissue that is solidified and sectioned.

\section{Spotting standard onto separate tissue section}

In the approach illustrated in Figure 7A, spots containing different concentrations of a standard are deposited directly onto a tissue section. MSI is used to generate an external calibration curve, which correlate the standard concentration to the detected signal intensity in the complex chemical matrix. Several studies have generated external standard curves of drugs on control tissue using MALDI-MSI then quantified the amount of drug present in tissue after drug administration.[49, $66,79,87]$ In these studies, the drug itself often is used as a standard as it is not present in the control tissue. For example, Hsieh et al. spotted different concentrations of a solution containing two standards, namely the drug clozapine and its metabolite norclozapine, on top of brain tissue to generate external calibration curves for both species using MALDI-MSI.[87] A similar approach was employed for quantifying the amount of tiotropium and imipramine in lung tissues after drug administration $[49,66]$ and evaluating the average concentration of two drugs in kidney tissue sections following dosing.[79] Despite the approach's success, it is difficult to generate a homogenous distribution of the standard in each spot due to a coffee-ring effect, which results in enhanced abundance of the standard at the edge of the spot.[38, 71, 89] To ensure high quality of the calibration spots in MALDI-MSI, Shroff et al. added fluorescent dye to the internal standard solution and determined the distribution of the standard in each spot using a fluorescence scanner.[90] Other studies have carefully selected the entire area of the spotted standard to get an average response, which helped compensate for the coffee-ring effect.[31]

\section{Response factors}

Instead of generating calibration curves, tissue-specific response factors often are introduced to compensate for varying matrix effects in different parts of the tissue and to facilitate 
quantification in Q-MSI.[45, 74, 84] To find the organ where the administered drugs localize, the tissue-specific response approach has been applied to whole body sections. In these experiments, internal standards are incorporated into the sample by soaking the tissue section in solution containing the standard prior to MSI. Tissue-specific ionization efficiency factors have been derived from MSI of whole body sections by accounting for the observed signal suppression in different organs.[45] Similarly, Hamm et al. introduced tissue extinction coefficient, or TEC, corresponding to signal intensity loss due to matrix effects in different organs of a whole body rat section.[84] After spraying standard solution on the sample, TEC is calculated using the intensity of the standard obtained on the tissue section divided by the intensity of the standard on the glass slide next to the tissue section. Dependence of TEC on the analyte concentration was examined by varying the standard's concentration. It was found that TEC does not depend on the standard's concentration, indicating that signal suppression is determined only by the composition of the complex mixture extracted from the tissue. To enable quantification, a calibration curve was generated by depositing standards onto the glass slide adjacent to the tissue. The drug concentration then was determined by dividing the average intensity of the drug obtained for each organ by the respective TEC.[84] Response factors also have been used for quantification in DESI-MSI. For example, Vismeh et al. deposited nine spots of loxapine calibration solution onto clozapine-treated rat brain tissue sections.[51] To minimize pixel-to-pixel variations, response factors were calculated using the ratio of clozapine to the standard. Quantification was performed by generating an external calibration curve.[51] Koeniger et al. introduced a different approach and defined a conversion factor to help correlate quantification using MALDI-MSI and LC-MS/MS.[74] In this approach, adjacent liver tissue sections were analyzed using both LC-MS/MS and MALDI-MSI after drug administration at different concentrations. Drug concentrations obtained with LC-MS/MS were used to find a conversion factor between the obtained MALDI-MSI intensity, in total ion counts per tissue sections and drug concentration in the tissue. Figure 8 shows representative quantitative ion images of the drug olanzapine in liver for each administered concentration.[74] Similar to tissue-specific ionization efficiency factors and TEC, the conversion factor is useful for quantifying average amounts of analyte in a tissue volume, such as an organ.

\section{Incorporating internal standard into a homogenized tissue}

Several groups have introduced tissue models to limit the amount of animal experiments required to explore the effect of drug dose on its accommodation in tissues. Typically, these models are generated using solidified tissue homogenates containing standards at different concentrations.[67, 73] Groseclose et al. added standards of the drug lapatinib into tissue homogenate that was subsequently frozen into a polymer support mold and cryo-sectioned.[67] Using MALDI-MSI, an external calibration curve was generated by examining tissue models containing different concentrations of the drug. The calibration curve then was applied to estimate lapatinib concentrations in a dosed animal tissue section.[67] Similarly, to generate an external calibration curve, Jadoul et al. added different amounts of deuterated PC to homogenized brain tissue.[73] This curve helped quantify endogenous PC species in a mouse brain tissue section using MALDI-MSI. Although the entire brain was homogenized in that study, the authors proposed that homogenates of particular regions of the brain could be used to better account for region-specific matrix effects when analyzing heterogeneous brain tissues.[73] Takai et al. used the combination of a tissue model and an internal standard deposition onto tissue sections for Q-MSI of a drug candidate 
in liver.[68] By depositing internal standard onto the model tissue, the study generated a calibration curve displaying the ratio of the analyte to the internal standard against the concentration of the standard. Afterward, MALDI-MSI was used to image thin tissue sections of multiple organs containing a uniformly deposited internal standard. Figures 9a-c show sections of the tissue model containing different concentrations of the drug candidate (9b) and the corresponding deuterated internal standard (9c). Figures 9d-f depict images of dosed kidney and liver containing analyte (9e) and internal standard (9f).[68]

\section{Online strategies}

Online quantification strategies rely on simultaneous detection of the internal standard and the extracted analyte. Several reports have shown that pixel-to-pixel signal variations are minimized by normalizing the analyte signal to that of the internal standard.[33, 35, 36, 42, 49-51, 53, 54, 65, 70, 71, 91-93] Furthermore, normalization to the internal standard signal observed in every pixel helps account for matrix effects occurring in individual ionization events during MSI experiments. Successful online quantification relies on the following assumptions: 1) the internal standard is present at equal concentrations in every spectrum, and 2) there is a close correspondence between the physiochemical properties of the internal standard and the analyte. The latter is achieved by using an isotopically labeled version of the analyte as an internal standard. Several strategies illustrated in Figure 10 have been employed to enable incorporation of internal standard(s) into QMSI experiments. These include (in Figure 10) A) coating the internal standard either on top or under the tissue section and B) incorporating internal standards into the extraction solvent.

\section{Coating with internal standard}

\section{On top of tissue}

A common on-line quantification strategy for MALDI-MSI involves depositing a uniform layer of an internal standard onto the tissue section in a similar manner as spraying the MALDI matrix. The internal standard can either be sprayed onto the tissue prior to spraying the MALDI matrix or incorporated in the MALDI matrix solution. Chumbley et al. evaluated different methods of applying standards for quantification in MALDI-MSI on tissues dosed with rifampicin (RIF).[31] Specifically, they used a robotic spotter to reproducibly apply an array of $\sim 200-\mu \mathrm{m}$ diameter RIF microspots onto both control tissue sections and tissues dosed with RIF in vitro and in vivo. To ensure adequate averaging over individual microspots, imaging experiments were performed with a lateral resolution of $200 \mu \mathrm{m}$. The most accurate results, in comparison with LC-MS/MS, were obtained by depositing the standard on top of the tissue followed by the matrix deposition. MALDI-MSI of control tissues prepared using the same protocol enabled quality control validation of Q-MSI.[31] Similarly, the pesticide chlordecone was quantified in mouse liver post administration by including an isotopically labeled internal standard into the MALDI matrix solution.[50] In a related study, Reyzer et al. included an internal standard into the MALDI matrix.[65] Källback et al. quantified endogenous Substance $P$ by spotting deuterated Substance $P$ onto the cortex of the brain where the signal from endogenous Substance $P$ was below the detection limit.[49] To quantify endogenous proteins in rat brain tissue sections, Clemis et al. sprayed a solution of MALDI matrix containing isotopically labeled peptides and trypsin onto the tissue section, which had been prewashed to remove lipids and metabolites.[92] Peptides produced by tryptic digestion of proteins were detected using MALDI-MSI. 
The intensity of the internal standard and endogenous peptide in each scan was used to construct a calibration curve and compensate for matrix effects in individual pixels.[92]

\section{Under tissue}

Q-MSI experiments also have been performed using an internal standard applied uniformly under the tissue section. Then, the internal standard is extracted into the matrix crystals in a similar manner as the analyte within the tissue. Pirman et al. used deuterated acetylcarnitine as an internal standard and applied it under the tissue section to quantify endogenous acetylcarnitine in the sample.[53, 88] A standard addition curve was generated by depositing acetylcarnitine at different concentrations onto the tissue section while accounting for matrix effects by normalizing to the deuterated internal standard.[53] Figure 11 shows the resulting ion images and standard curves for acetylcarnitine. Figure 11A displays the ion image of acetylcarnitine, illustrating both the endogenous and deposited standard at $\mathrm{m} / \mathrm{z}$ 204. In Figure 11B, the ion images of deuterated acetylcarnitine display matrix effects originating from the chemical heterogeneity of the tissue section. Figure $11 \mathrm{C}$ shows the ion image obtained by normalizing the signal of acetylcarnitine to deuterated acetylcarnitine. Normalization to the deuterated standards minimized standard deviations in the calibration curves (shown in Figures 11D and 11E).[53, 70] Bokhart et al. quantified the HIV drug emtricitabine in cervical tissue using MALDESI by generating an external calibration curve of deposited isotopically labeled emtricitabine on top of the tissue section and lamivudine as an internal standard under the tissue.[71] Landgraf et al. quantified endogenous PC in tissue in each pixel using MALDI-MSI by uniformly depositing an internal standard under the tissue.[85] The amount of the observed analyte in moles/pixel was calculated using Eq. 1.

$$
\text { Moles }=\frac{I n t_{A N}}{\operatorname{Int}_{I S}} \times \frac{W_{I S}}{M W_{A N}} \times A
$$

Int $t_{A N}$ is the analyte signal intensity, Int $/$ is the intensity of the internal standard, $W_{I S}$ is the amount of the internal standard deposited onto the glass slide, $M W_{A N}$ is the analyte's molecular weight, and $A$ is the area of the tissue.[85]

\section{Q-MSI using ambient liquid extraction techniques}

Q-MSI studies using liquid extraction techniques have the ability to incorporate the internal standard directly into the extraction solvent. This approach ensures that the internal standard is present at a constant concentration throughout an imaging experiment. By including a known concentration of deuterated nicotine into the nano-DESI solvent, Lanekoff et al. quantified nicotine in rat brain after administration.[33] This approach used the intensity ratio of nicotine to deuterated nicotine to calculate the amount nicotine detected in each pixel, according to Eq. 2.

amount nicotine $\left(\frac{\text { moles }}{\text { pixel }}\right)=C_{\text {std }} \times \frac{I_{\text {nicotine }}}{I_{\text {std }}} \times$ Flowrate $_{\text {std }} \times I T$

$I_{\text {nicotine }}$ is the intensity of nicotine, $I_{\text {std }}$ is the intensity of the deuterated nicotine used as an internal standard, $C_{\text {std }}$ is the concentration of deuterated nicotine, Flowrate std $_{\text {is }}$ is the solvent flow rate, and $I T$ is the ion accumulation time for each scan in the Orbitrap.[33] In a related study, Lanekoff et al. developed a shotgun-like quantification approach for Q-MSI of all endogenous PC in rat brain tissue section using only two PC standards added to the nano-DESI solvent. The approach used a modified version of Eq. 2 that accounts for carbon factors representing differences in ion suppression 
stemming from differences in acyl chain length.[43] Carbon factors were determined using two PC standards, one with shorter and another with longer acyl chain lengths. In addition, the extraction efficiency of endogenous PC species from the sample surface was investigated and discovered to be consistent between different regions of the rat brain tissue section.[43] Figure 12A shows quantitative ion images of four different PC species, namely PC 32:0, PC 34:1, PC 36:4, and PC 36:1 with their individual intensity scales in fmoles/pixel. Figure $12 \mathrm{C}$ depicts the quantitative data represented by bar graphs from specific regions of interest defined in Figure 12B. In a related study, nano-DESI MSI was used to quantify three small neurotransmitters in a rat brain tissue section simultaneously by incorporating three deuterated internal standards into the nano-DESI solvent.[36] Internal standards also have been incorporated into the extraction solvent for quantitative analysis using LESA.[52, 94] Parson et al. added deuterated chloroquine into LESA solvent and spatially profiled the amount of chloroquine in the kidneys of dosed rats.[52] Figure 13 shows that the highest concentration of chloroquine was found in the renal medulla. By also including deuterated internal standards of chloroquine metabolites in the solvent, the study followed chloroquine metabolism over a $24 \mathrm{~h}$ time course.[52, 95]

\section{Future Directions}

Further development and validation of Q-MSI are needed to ensure its accuracy, robust operation, and applicability to a broad range of compounds. By adding several types of standards, it may be possible to obtain quantitative images of a large variety of endogenous species present in the sample. Several aspects affect Q-MSI accuracy, including sample preparation, pixel-to-pixel signal stability, and analyte extraction efficiency. Signal variability in MSI may be detrimental to accurate quantification. A recent interlaboratory study examined the repeatability and constancy of DESI signal intensities obtained for the same reference samples across 20 laboratories.[96] Although repeatability of better than 20 percent was obtained by several groups, imperfections in the source design have been shown to substantially degrade DESI performance. Similar repeatability of $\sim 15-20$ percent is commonly achieved in nano-DESI MSI experiments.[23] Shot-to-shot variations in MALDI experiments also may be reduced by improving matrix deposition approaches. For example, higher reproducibility has been obtained when small, evenly distributed matrix crystals are deposited onto the sample surface.[97] Substantial efforts have been dedicated to the optimization of matrix application approaches for MALDI-MSI.[98] For example, sublimation of the matrix on top of tissue followed by sample rehydration has been identified as the best matrix application method for highresolution imaging.[99] However, less efficient extraction of analytes reduces sensitivity and may not be ideal for Q-MSI experiments. Furthermore, possible delocalization of analytes during sample rehydration is a concern.[100] Robotic spraying of matrix solution onto a sample is used by many groups and has been a viable approach for high-resolution imaging. Again, when using this approach, care should be taken to avoid delocalization of analytes. Moreover, signal repeatability in MALDI may be affected by laser ablation efficiency, which is controlled by adjusting the number of laser shots.[101] It is anticipated that further optimization of matrix application approaches also will improve shot-to-shot repeatability of MALDI signals, which will greatly benefit Q-MSI experiments.

Analyte extraction efficiency is another important factor affecting Q-MSI accuracy. As discussed, analyte extraction in MALDI is affected by the matrix application approach and the volume of ablated material controlled by the number of laser shots. It is known that tissue composition also may affect analyte extraction efficiency in MALDI.[98] Similarly, tissue composition could affect the 
extraction efficiency in DESI and direct liquid extraction ambient ionization approaches. Although similar extraction efficiency has been observed from the white and gray matter in nano-DESI MSI of rat brain tissue sections,[54] these observations cannot be extrapolated to other types of tissues. It follows that additional experiments are necessary to characterize the dependence of extraction efficiency on the tissue composition in Q-MSI experiments.

In summary, Q-MSI is a powerful technique for mapping chemical gradients in complex biological systems. Better understanding of the processes relevant to Q-MSI will assist in developing approaches for robust quantitative analysis of molecules in chemically complex biological samples. Compared to bulk analysis after tissue homogenization, MSI enables quantitative analysis of molecules present in relatively small regions of tissue sections, which may be difficult to detect in tissue homogenates. Q-MSI requires a targeted approach and relies on using carefully selected internal standards to account for tissue-specific matrix effects. The best strategy involves using isotopically labeled internal standards and normalizing the intensity of the analyte to the intensity of the internal standard in the same pixel to account for pixel-specific matrix effects. Shotgun-like quantification of lipids also can be performed using only two standards from the same lipid class. This approach can be readily expanded to other biomolecules and used for simultaneous Q-MSI of multiple analyte classes. Future advancements in the development and validation of robust Q-MSI approaches will establish this technique as a transformational tool for studies in biology, drug discovery, and clinical research.

\section{REFERENCES}

1. Koestler, M., et al., A high-resolution scanning microprobe matrix-assisted laser desorption/ionization ion source for imaging analysis on an ion trap/Fourier transform ion cyclotron resonance mass spectrometer. Rapid Communications in Mass Spectrometry, 2008. 22(20): p. 3275-3285.

2. Römpp, A. and B. Spengler, Mass spectrometry imaging with high resolution in mass and space. Histochemistry and Cell Biology, 2013. 139(6): p. 759-783.

3. Sampson, J.S., A.M. Hawkridge, and D.C. Muddiman, Generation and detection of multiplycharged peptides and proteins by matrix-assisted laser desorption electrospray ionization (MALDESI) fourier transform ion cyclotron resonance mass spectrometry. Journal of the American Society for Mass Spectrometry, 2006. 17(12): p. 1712-1716.

4. Cooks, R.G., et al., Ambient Mass Spectrometry. Science, 2006. 311(5767): p. 1566-1570.

5. Weston, D.J., Ambient ionization mass spectrometry: current understanding of mechanistic theory; analytical performance and application areas. Analyst, 2010. 135(4): p. 661-668.

6. $\mathrm{Wu}, \mathrm{C}$., et al., Mass spectrometry imaging under ambient conditions. Mass Spectrometry Reviews, 2013. 32(3): p. 218-243.

7. Monge, M.E., et al., Mass Spectrometry: Recent Advances in Direct Open Air Surface Sampling/lonization. Chemical Reviews, 2013. 113(4): p. 2269-2308.

8. Badu-Tawiah, A.K., et al., Chemical Aspects of the Extractive Methods of Ambient Ionization Mass Spectrometry. Annual Review of Physical Chemistry, 2013. 64(1): p. 481-505.

9. Ifa, D.R., et al., Desorption electrospray ionization and other ambient ionization methods: current progress and preview. Analyst, 2010. 135(4): p. 669-681.

10. Van Berkel, G.J., S.P. Pasilis, and O. Ovchinnikova, Established and emerging atmospheric pressure surface sampling/ionization techniques for mass spectrometry. Journal of Mass Spectrometry, 2008. 43(9): p. 1161-1180. 
11. Hsu, C.-C. and P.C. Dorrestein, Visualizing life with ambient mass spectrometry. Current Opinion in Biotechnology, 2015. 31: p. 24-34.

12. Venter, A.R., et al., Mechanisms of Real-Time, Proximal Sample Processing during Ambient Ionization Mass Spectrometry. Analytical Chemistry, 2014. 86(1): p. 233-249.

13. Laskin, J. and I. Lanekoff, Ambient Mass Spectrometry Imaging Using Direct Liquid Extraction Techniques. Analytical Chemistry, 2016. 88(1): p. 52-73.

14. Campbell, D.I., et al., Improved spatial resolution in the imaging of biological tissue using desorption electrospray ionization. Analytical and Bioanalytical Chemistry, 2012. 404(2): p. 389-398.

15. Kertesz, V. and G.J. Van Berkel, Fully automated liquid extraction-based surface sampling and ionization using a chip-based robotic nanoelectrospray platform. Journal of Mass Spectrometry, 2010. 45(3): p. 252-260.

16. Roach, P.J., J. Laskin, and A. Laskin, Nanospray desorption electrospray ionization: an ambient method for liquid-extraction surface sampling in mass spectrometry. Analyst, 2010. 135(9): p. 2233-2236.

17. Swales, J.G., et al., Anal. Chem., 2014. 86(16): p. 8473.

18. Brenton, A.G. and A.R. Godfrey, Electro-Focusing Liquid Extractive Surface Analysis (EF-LESA) Coupled to Mass Spectrometry. Analytical Chemistry, 2014. 86(7): p. 3323-3329.

19. Abraham, J.L., S. Chandra, and A. Agrawal, Quantification and micron-scale imaging of spatial distribution of trace beryllium in shrapnel fragments and metallurgic samples with correlative fluorescence detection method and secondary ion mass spectrometry (SIMS). Journal of Microscopy, 2014. 256(2): p. 145-152.

20. Lanekoff, I., et al., Three-dimensional imaging of lipids and metabolites in tissues by nanospray desorption electrospray ionization mass spectrometry. Analytical and Bioanalytical Chemistry, 2015. 407(8): p. 2063-2071.

21. Lanekoff, I., et al., Automated Platform for High-Resolution Tissue Imaging Using Nanospray Desorption Electrospray Ionization Mass Spectrometry. Analytical Chemistry, 2012. 84(19): p. 8351-8356.

22. Lanekoff, I. and J. Laskin, Imaging of lipids and metabolites using nanospray desorption electrospray ionization mass spectrometry. Methods in molecular biology (Clifton, N.J.), 2015. 1203: p. 99-106.

23. Laskin, J., et al., Tissue Imaging Using Nanospray Desorption Electrospray Ionization Mass Spectrometry. Analytical Chemistry, 2012. 84(1): p. 141-148.

24. Annesley, T.M., Ion suppression in mass spectrometry. Clinical Chemistry, 2003. 49(7): p. 1041-1044.

25. Knochenmuss, R., et al., The Matrix Suppression Effect and lonization Mechanisms in Matrixassisted Laser Desorption/Ionization. Rapid Communications in Mass Spectrometry, 1996. 10(8): p. 871-877.

26. Ellis, S.R., A.L. Bruinen, and R.M.A. Heeren, A critical evaluation of the current state-of-the-art in quantitative imaging mass spectrometry. Analytical and Bioanalytical Chemistry, 2014. 406(5): p. 1275-1289.

27. Lietz, C.B., E. Gemperline, and L. Li, Qualitative and quantitative mass spectrometry imaging of drugs and metabolites. Advanced Drug Delivery Reviews, 2013. 65(8): p. 1074-1085.

28. Prideaux, B. and M. Stoeckli, J. Proteomics, 2012. 75: p. 4999.

29. Castellino, S., M.R. Groseclose, and D. Wagner, MALDI imaging mass spectrometry: bridging biology and chemistry in drug development. Bioanalysis, 2011. 3(21): p. 2427-2441.

30. Taylor, P.J., Matrix effects: The Achilles heel of quantitative high-performance liquid chromatography-electrospray-tandem mass spectrometry. Clinical Biochemistry, 2005. 38(4): p. 328-334.

31. Chumbley, C.W., et al., Absolute Quantitative MALDI Imaging Mass Spectrometry: A Case of Rifampicin in Liver Tissues. Analytical Chemistry, 2016. 
32. Dong, Y., G. Guella, and P. Franceschi, Impact of tissue surface properties on the desorption electrospray ionization imaging of organic acids in grapevine stem. Rapid Communications in Mass Spectrometry, 2016. 30(6): p. 711-718.

33. Lanekoff, I., et al., Imaging Nicotine in Rat Brain Tissue by Use of Nanospray Desorption Electrospray Ionization Mass Spectrometry. Analytical Chemistry, 2012. 85(2): p. 882-889.

34. Muramoto, S., et al., Test Sample for the Spatially Resolved Quantification of Illicit Drugs on Fingerprints Using Imaging Mass Spectrometry. Analytical Chemistry, 2015. 87(10): p. 54445450.

35. Park, K.M., et al., Relative Quantification in Imaging of a Peptide on a Mouse Brain Tissue by Matrix-Assisted Laser Desorption Ionization. Analytical Chemistry, 2014. 86(10): p. 51315135.

36. Bergman, H.-M., et al., Quantitative mass spectrometry imaging of small-molecule neurotransmitters in rat brain tissue sections using nanospray desorption electrospray ionization. Analyst, 2016.

37. Porta, T., et al., Quantification in MALDI-MS imaging: what can we learn from MALDIselected reaction monitoring and what can we expect for imaging? Analytical and Bioanalytical Chemistry, 2015. 407(8): p. 2177-2187.

38. Reich, R.F., et al., Quantitative MALDI-MSn Analysis of Cocaine in the Autopsied Brain of a Human Cocaine User Employing a Wide Isolation Window and Internal Standards. Journal of the American Society for Mass Spectrometry, 2010. 21(4): p. 564-571.

39. Rosen, E.P., et al., Influence of Desorption Conditions on Analyte Sensitivity and Internal Energy in Discrete Tissue or Whole Body Imaging by IR-MALDESI. Journal of the American Society for Mass Spectrometry, 2015. 26(6): p. 899-910.

40. Sugiyama, E., et al., Ammonium Sulfate Improves Detection of Hydrophilic Quaternary Ammonium Compounds through Decreased Ion Suppression in Matrix-Assisted Laser Desorption/Ionization Imaging Mass Spectrometry. Analytical Chemistry, 2015. 87(22): p. 11176-11181.

41. Tomlinson, L., et al., Using a single, high mass resolution mass spectrometry platform to investigate ion suppression effects observed during tissue imaging. Rapid Communications in Mass Spectrometry, 2014. 28(9): p. 995-1003.

42. Lanekoff, I., et al., Matrix effects in biological mass spectrometry imaging: identification and compensation. Analyst, 2014. 139(14): p. 3528-3532.

43. Lanekoff, I., M. Thomas, and J. Laskin, Shotgun Approach for Quantitative Imaging of Phospholipids Using Nanospray Desorption Electrospray Ionization Mass Spectrometry. Analytical Chemistry, 2014.

44. Prideaux, B., et al., High-Sensitivity MALDI-MRM-MS Imaging of Moxifloxacin Distribution in Tuberculosis-Infected Rabbit Lungs and Granulomatous Lesions. Analytical Chemistry, 2011. 83(6): p. 2112-2118.

45. Stoeckli, M., D. Staab, and A. Schweitzer, Compound and metabolite distribution measured by MALDI mass spectrometric imaging in whole-body tissue sections. Int. J. Mass Spectrom., 2007. 260(2-3): p. 195-202.

46. Hochart, G., G. Hamm, and J. Stauber, Label-free MS imaging from drug discovery to preclinical development. Bioanalysis, 2014. 6(20): p. 2775-2788.

47. Prideaux, B., et al., Mass spectrometry imaging of levofloxacin distribution in TB-infected pulmonary lesions by MALDI-MSI and continuous liquid microjunction surface sampling. International Journal of Mass Spectrometry, 2015. 377: p. 699-708.

48. Yunsheng, H., et al., Visualization of First-Pass Drug Metabolism of Terfenadine by MALDIImaging Mass Spectrometry. Drug Metabolism Letters, 2008. 2(1): p. 1-4.

49. Kallback, P., et al., Novel mass spectrometry imaging software assisting labeled normalization and quantitation of drugs and neuropeptides directly in tissue sections. Journal of Proteomics, 2012. 75(16): p. 4941-4951. 
50. Lagarrigue, M., et al., Localization and in Situ Absolute Quantification of Chlordecone in the Mouse Liver by MALDI Imaging. Analytical Chemistry, 2014. 86(12): p. 5775-5783.

51. Vismeh, R., et al., Localization and quantification of drugs in animal tissues by use of desorption electrospray ionization mass spectrometry imaging. Anal. Chem., 2012. 84(12): p. 5439-45.

52. Parson, W.B., et al., J. Mass Spectrom., 2012. 47: p. 1420.

53. Pirman, D.A., et al., Identifying Tissue-Specific Signal Variation in MALDI Mass Spectrometric Imaging by Use of an Internal Standard. Analytical Chemistry, 2013. 85(2): p. 1090-1096.

54. Lanekoff, I., M. Thomas, and J. Laskin, Shotgun Approach for Quantitative Imaging of Phospholipids Using Nanospray Desorption Electrospray Ionization Mass Spectrometry. Analytical Chemistry, 2014. 86(3): p. 1872-1880.

55. Hankin, J.A. and R.C. Murphy, Relationship between MALDI IMS Intensity and Measured Quantity of Selected Phospholipids in Rat Brain Sections. Analytical Chemistry, 2010. 82(20): p. 8476-8484.

56. Cerruti, C.D., et al., Anal. Bioanal. Chem., 2011. 401: p. 75.

57. Wang, H.-Y.J., et al., MALDI-mass spectrometry imaging of desalted rat brain sections reveals ischemia-mediated changes of lipids. Analytical and Bioanalytical Chemistry, 2012. 404(1): p. 113-124.

58. Wang, H.-Y.J., C.B. Liu, and H.-W. Wu, A simple desalting method for direct MALDI mass spectrometry profiling of tissue lipids. Journal of Lipid Research, 2011. 52(4): p. 840-849.

59. Goodwin, R.J.A., et al., Rapid Commun. Mass Spectrom., 2010. 24: p. 1682.

60. Sun, N. and A. Walch, Qualitative and quantitative mass spectrometry imaging of drugs and metabolites in tissue at therapeutic levels. Histochemistry and Cell Biology, 2013. 140(2): p. 93-104.

61. Goodwin, R.J.A., et al., Anal. Chem., 2010. 82: p. 3868.

62. Nilsson, A., et al., Mass Spectrometry Imaging in Drug Development. Analytical Chemistry, 2015. 87(3): p. 1437-1455.

63. Greer, T., R. Sturm, and L. Li, Mass spectrometry imaging for drugs and metabolites. J. Proteomics, 2011. 74(12): p. 2617-2631.

64. Marsching, C., et al., Quantitative imaging mass spectrometry of renal sulfatides: validation by classical mass spectrometric methods. Journal of Lipid Research, 2014. 55(11): p. 23432353.

65. Reyzer, M.L., et al., J. Mass Spectrom., 2003. 38: p. 1081.

66. Nilsson, A., et al., Fine Mapping the Spatial Distribution and Concentration of Unlabeled Drugs within Tissue Micro-Compartments Using Imaging Mass Spectrometry. Plos One, 2010. 5(7).

67. Groseclose, M.R. and S. Castellino, A Mimetic Tissue Model for the Quantification of Drug Distributions by MALDI Imaging Mass Spectrometry. Analytical Chemistry, 2013. 85(21): p. 10099-10106.

68. Takai, N., Y. Tanaka, and H. Saji, Quantification of small molecule drugs in biological tissue sections by imaging mass spectrometry using surrogate tissue-based calibration standards. Mass spectrometry (Tokyo, Japan), 2014. 3(1): p. A0025-A0025.

69. Schulz, S., et al., DMSO-enhanced MALDI MS imaging with normalization against a deuterated standard for relative quantification of dasatinib in serial mouse pharmacology studies. Analytical and Bioanalytical Chemistry, 2013. 405(29): p. 9467-9476.

70. Pirman, D.A., et al., Quantitative MALDI Tandem Mass Spectrometric Imaging of Cocaine from Brain Tissue with a Deuterated Internal Standard. Analytical Chemistry, 2013. 85(2): p. 1081-1089.

71. Bokhart, M.T., et al., Quantitative mass spectrometry imaging of emtricitabine in cervical tissue model using infrared matrix-assisted laser desorption electrospray ionization. Analytical and Bioanalytical Chemistry, 2015. 407(8): p. 2073-2084. 
72. Porta, T., et al., Single Hair Cocaine Consumption Monitoring by Mass Spectrometric Imaging. Analytical Chemistry, 2011. 83(11): p. 4266-4272.

73. Jadoul, L., et al., A spiked tissue-based approach for quantification of phosphatidylcholines in brain section by MALDI mass spectrometry imaging. Analytical and Bioanalytical Chemistry, 2015. 407(8): p. 2095-2106.

74. Koeniger, S.L., et al., $A$ quantitation method for mass spectrometry imaging. Rapid Communications in Mass Spectrometry, 2011. 25(4): p. 503-510.

75. Takai, N., et al., Quantitative analysis of pharmaceutical drug distribution in multiple organs by imaging mass spectrometry. Rapid Communications in Mass Spectrometry, 2012. 26(13): p. $1549-1556$.

76. Bocklitz, T.W., et al., Deeper Understanding of Biological Tissue: Quantitative Correlation of MALDI-TOF and Raman Imaging. Analytical Chemistry, 2013. 85(22): p. 10829-10834.

77. Forest, S., et al., Surface Plasmon Resonance Imaging-MALDI-TOF Imaging Mass Spectrometry of Thin Tissue Sections. Analytical Chemistry, 2016.

78. Aichler, M., et al., Spatially Resolved Quantification of Gadolinium(III)-Based Magnetic Resonance Agents in Tissue by MALDI Imaging Mass Spectrometry after In Vivo MRI. Angewandte Chemie-International Edition, 2015. 54(14): p. 4279-4283.

79. Goodwin, R.J.A., et al., Qualitative and Quantitative MALDI Imaging of the Positron Emission Tomography Ligands Raclopride (a D2 Dopamine Antagonist) and SCH 23390 (a D1 Dopamine Antagonist) in Rat Brain Tissue Sections Using a Solvent-Free Dry Matrix Application Method. Analytical Chemistry, 2011. 83(24): p. 9694-9701.

80. Drexler, D.M., et al., Utility of quantitative whole-body autoradiography (QWBA) and imaging mass spectrometry (IMS) by matrix-assisted laser desorption/ionization (MALDI) in the assessment of ocular distribution of drugs. Journal of Pharmacological and Toxicological Methods, 2011. 63(2): p. 205-208.

81. Solon, E.G., et al., AAPS J., 2010. 12: p. 11.

82. Schadt, S., et al., Investigation of Figopitant and Its Metabolites in Rat Tissue by Combining Whole-Body Autoradiography with Liquid Extraction Surface Analysis Mass Spectrometry. Drug Metabolism and Disposition, 2012. 40(3): p. 419-425.

83. Goodwin, R.J.A., Sample preparation for mass spectrometry imaging: Small mistakes can lead to big consequences. Journal of Proteomics, 2012. 75(16): p. 4893-4911.

84. Hamm, G., et al., J. Proteomics, 2012. 75: p. 4952.

85. Landgraf, R.R., et al., Considerations for quantification of lipids in nerve tissue using matrixassisted laser desorption/ionization mass spectrometric imaging. Rapid Communications in Mass Spectrometry, 2011. 25(20): p. 3178-3184.

86. Takai, N., et al., Quantitative imaging of a therapeutic peptide in biological tissue sections by MALDI MS. Bioanalysis, 2013. 5(5): p. 603-612.

87. Hsieh, Y., et al., Matrix-assisted laser desorption/ionization imaging mass spectrometry for direct measurement of clozapine in rat brain tissue. Rapid Communications in Mass Spectrometry, 2006. 20(6): p. 965-972.

88. Pirman, D.A. and R.A. Yost, Quantitative Tandem Mass Spectrometric Imaging of Endogenous Acetyl-L-carnitine from Piglet Brain Tissue Using an Internal Standard. Analytical Chemistry, 2011. 83(22): p. 8575-8581.

89. Liu, X. and A.B. Hummon, Mass Spectrometry Imaging of Therapeutics from Animal Models to Three-Dimensional Cell Cultures. Analytical Chemistry, 2015. 87(19): p. 9508-9519.

90. Shroff, R., et al., Quantification of plant surface metabolites by matrix-assisted laser desorption-ionization mass spectrometry imaging: glucosinolates on Arabidopsis thaliana leaves. Plant Journal, 2015. 81(6): p. 961-972.

91. Bunch, J., M.R. Clench, and D.S. Richards, Rapid Commun. Mass Spectrom., 2004. 18: p. 3051. 92. Clemis, E.J., et al., Quantitation of Spatially-Localized Proteins in Tissue Samples using MALDIMRM Imaging. Analytical Chemistry, 2012. 84(8): p. 3514-3522. 
93. Lee, Y.J., et al., Use of mass spectrometry for imaging metabolites in plants. Plant Journal, 2012. 70(1): p. 81-95.

94. Almeida, R., et al., Quantitative Spatial Analysis of the Mouse Brain Lipidome by Pressurized Liquid Extraction Surface Analysis. Analytical Chemistry, 2015. 87(3): p. 1749-1756.

95. Mandal, M.K., et al., Development of Sheath-Flow Probe Electrospray Ionization Mass Spectrometry and Its Application to Real Time Pesticide Analysis. Journal of Agricultural and Food Chemistry, 2013. 61(33): p. 7889-7895.

96. Gurdak, E., et al., VAMAS Interlaboratory Study for Desorption Electrospray Ionization Mass Spectrometry (DESI MS) Intensity Repeatability and Constancy. Analytical Chemistry, 2014. 86(19): p. 9603-9611.

97. Önnerfjord, P., et al., Homogeneous sample preparation for automated high throughput analysis with matrix-assisted laser desorption/ionisation time-of-flight mass spectrometry. Rapid Communications in Mass Spectrometry, 1999. 13(5): p. 315-322.

98. Gessel, M.M., J.L. Norris, and R.M. Caprioli, MALDI imaging mass spectrometry: Spatial molecular analysis to enable a new age of discovery. Journal of Proteomics, 2014. 107: p. 7182.

99. Hankin, J.A., R.M. Barkley, and R.C. Murphy, Sublimation as a Method of Matrix Application for Mass Spectrometric Imaging. Journal of the American Society for Mass Spectrometry, 2007. 18(9): p. 1646-1652.

100. Yang, J. and R.M. Caprioli, Matrix Sublimation/Recrystallization for Imaging Proteins by Mass Spectrometry at High Spatial Resolution. Analytical Chemistry, 2011. 83(14): p. 5728-5734.

101. Robichaud, G., et al., Infrared Matrix-Assisted Laser Desorption Electrospray Ionization (IRMALDESI) Imaging Source Coupled to a FT-ICR Mass Spectrometer. Journal of The American Society for Mass Spectrometry, 2012. 24(1): p. 92-100.

\section{FIGURES}

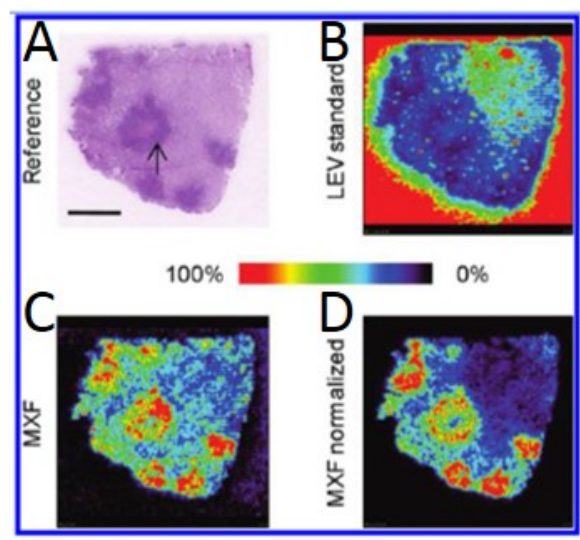

Figure 1. Figure showing ion images obtained by MALDI-MSI at each stage of the normalization process: (A) H\&E reference image, (B) ion image of levofloxacin (LEV) standard, and (C) ion image of administered moxifloxacin (MXF). In (D), MXF normalized to LEV signals are observed from the granulomas (large central granuloma, indicated by the arrow in the H\&E image) compared to that of the surrounding normal lung tissue. Greater LEV signal suppression occurred in the viable granuloma compared to that in the caseum, identifiable as the light pink center of the large central granuloma. Ion signal intensities were individually scaled for each image. Scale bar $=5 \mathrm{~mm}$. Reprinted with permission from Prideaux, B.; Dartois, V.; Staab, D.; Weiner, D. M.; Goh, A.; Via, L. E.; Barry III, C. E.; 
Stoeckli, M., High-Sensitivity MALDI-MRM-MS Imaging of Moxifloxacin Distribution in TuberculosisInfected Rabbit Lungs and Granulomatous Lesions. Analytical Chemistry, 2011, 83, 2112-2118 (Ref [44]). Copyright (2011) American Chemical Society.
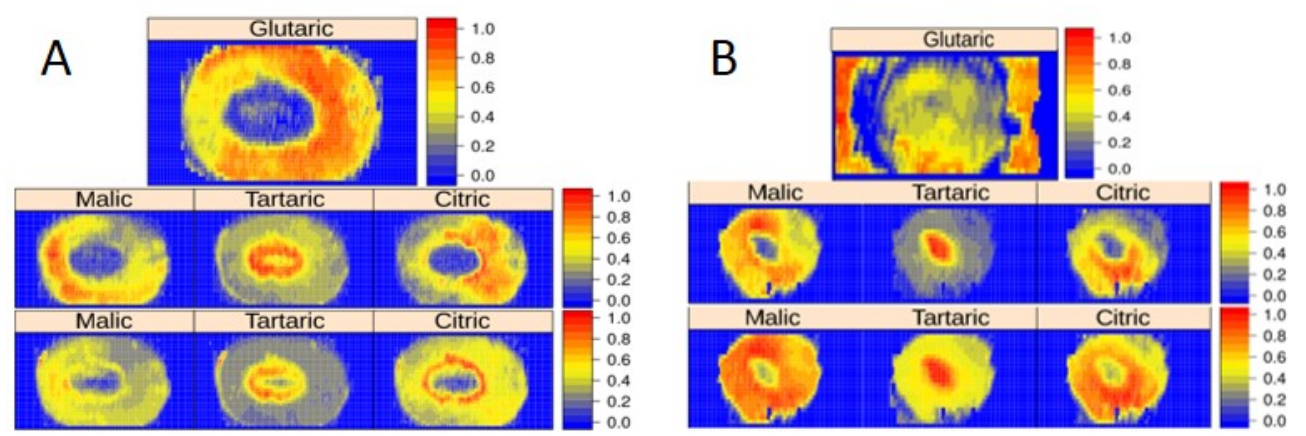

Figure 2. DESI-MSI of organic acids in grapevine stem using glutaric acid as the internal standard. Top rows show the ion image of the internal standard, middle rows feature ion images of three organic acids, and the bottom rows depict the ion images of the organic acids normalized to the internal standard. In (A), the stem was soaked in the internal standard prior to MSI, and, in (B), the internal standard was included in the DESI solvent during MSI. Used with permission from Dong, Y.; Guella, G.; Franceschi, P., Impact of tissue surface properties on the desorption electrospray ionization imaging of organic acids in grapevine stem. Rapid Communication in Mass Spectrometry, 2016, 30, 711-718 (Ref [32]). Wiley.

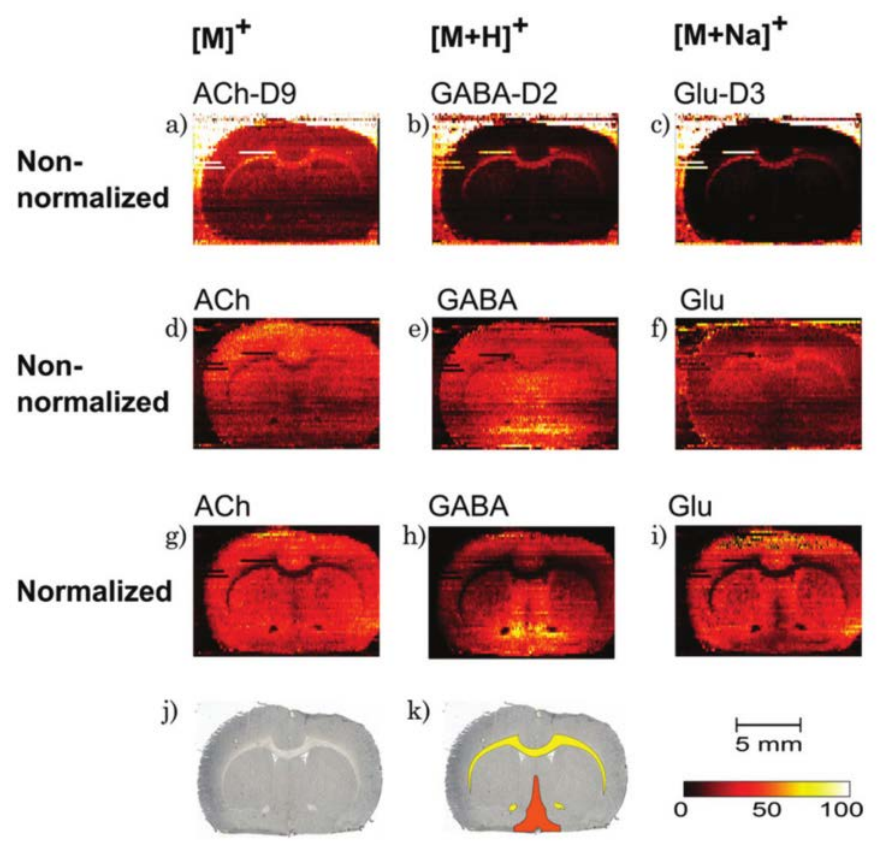

Figure 3. Ion images of $A C h, G A B A$, and Glu in a rat brain tissue section. (a) Non-normalized ion image of $\left[A C h-D_{9}\right]^{+}$, (b) non-normalized ion image of $\left[G A B A-D_{2}+H\right]^{+}$, (c) non-normalized ion image of [Glu$\left.\mathrm{D}_{3}+\mathrm{Na}\right]^{+}$, (d) non-normalized ion image of endogenous $[\mathrm{ACh}]^{+}$, (e) non-normalized ion image of endogenous $[\mathrm{GABA}+\mathrm{H}]^{+}$, (f) non-normalized ion image of endogenous $[\mathrm{Glu}+\mathrm{Na}]^{+}$, (g) normalized ion image of $[\mathrm{ACh}]^{+}$, (h) normalized ion image of $[\mathrm{GABA}+\mathrm{H}]^{+}$, (i) normalized ion image of [Glu $\left.+\mathrm{Na}\right]^{+},(\mathrm{j})$ optical image of the analyzed brain tissue section, (k) optical image of analyzed brain tissue section 
with white matter regions highlighted in yellow and the medial septum-diagonal band complex highlighted in orange. Scale bar: $5 \mathrm{~mm}$. The signal intensity of the ion images scale from dark to bright. The figure is reproduced from Bergman, H-M.; Lundin, E.; Andersson, M.; Lanekoff, I., Quantitative mass spectrometry imaging of small-molecule neurotransmitters in rat brain tissue sections using nanospray desorption electrospray ionization. Analyst 2016 (Ref [36]). Reproduced by permission of The Royal Society of Chemistry.
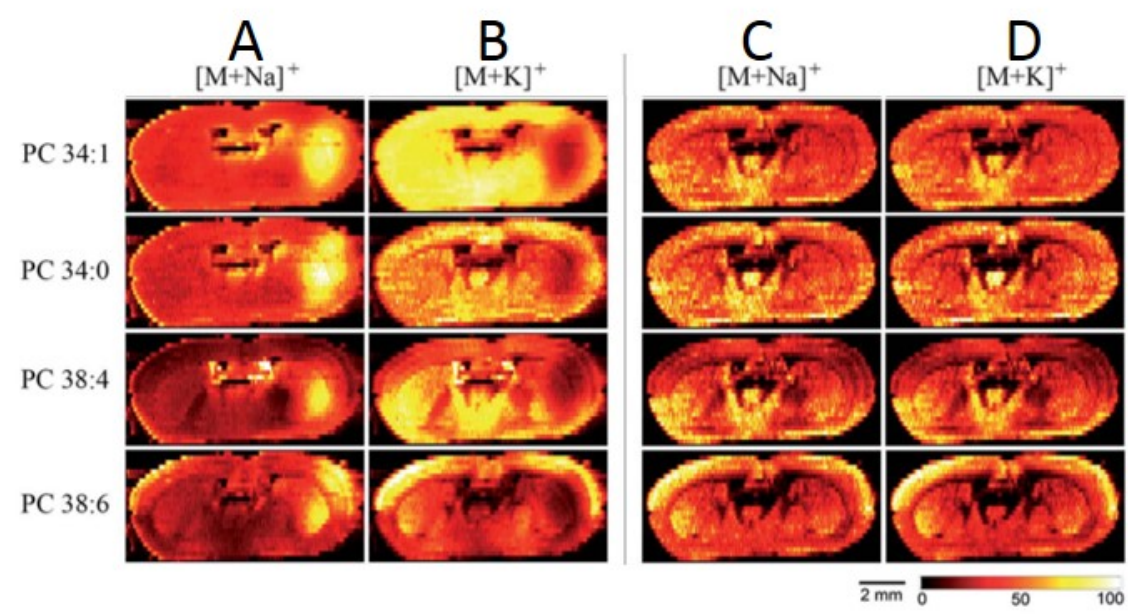

Figure 4. Ion images of sodium and potassium adducts, $[\mathrm{M}+\mathrm{Na}]^{+}$and $[\mathrm{M}+\mathrm{K}]^{+}$, of endogenous $\mathrm{PC}$ 34:1, PC 34:0, PC 38:4, and PC 38:6. Ion images are normalized to the total ion current (TIC) in columns $A$ and $B$ and to the internal standard PC 25:0 in columns $C$ and $D$. Lateral scale bar is $2 \mathrm{~mm}$. Intensity scale bar ranges from 0 (black) to $100 \%$ (light yellow) signal intensity of an individual peak. Figure reproduced from Lanekoff, I.; Stevens, S. L.; Stenzel-Poore, M. P.; Laskin, J., Matrix effects in biological mass spectrometry imaging: identification and compensation. Analyst 2014, 139, 35283532 (Ref [42]). Reproduced by permission of The Royal Society of Chemistry.
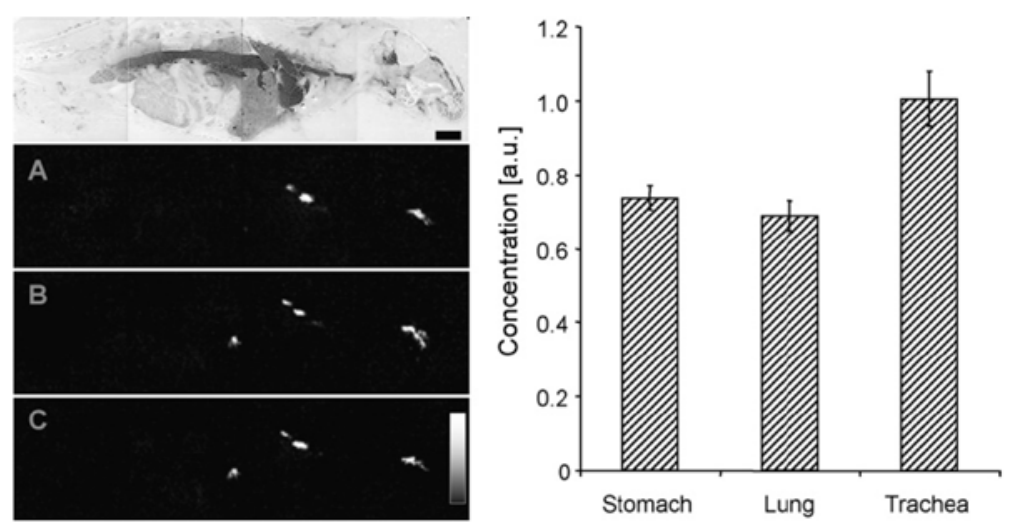

Figure 5. Relative quantification by MSI. Left: optical image (top) and MSI images from three different sections of the same animal $(A-C)$. Data evaluation was obtained by averaging pixel intensities over the respective tissues. Error bars for the three samples appear at right. Reprinted from Stoeckli, M.; Staab D.; Schweitzer, A., Compound and metabolite distribution measured by MALDI mass spectrometric imaging in whole-body tissue sections. International Journal of Mass Spectrometry 2007, 260, 195-202 (Ref [45]). Copyright (2007) with permission from Elsevier. 

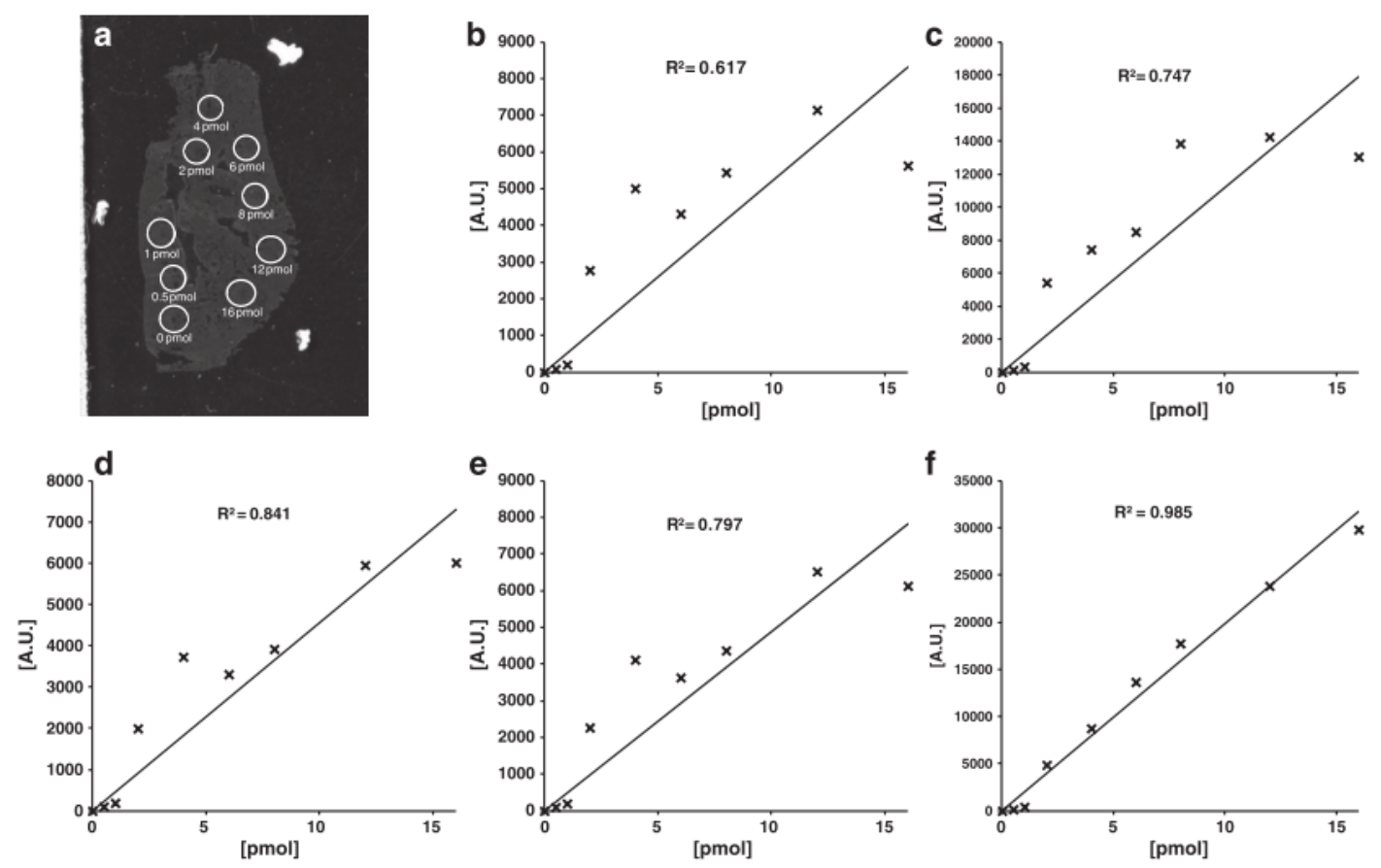

Figure 6. Calibration curve plots of imipramine standards using different normalization methods. a) Digital photograph of a lung tissue section shown with different amounts of imipramine (blank, 0.5, $1,2,4,6,8,12$, and $16 \mathrm{pmol}$ in $0.2 \mu \mathrm{l}$ ) applied on a rat lung tissue section. The average diameter of the spots was calculated to $1.51 \mathrm{~mm}$. The correlation coefficients $\left(R^{2}\right)$ for the concentration standards were calculated using different normalization methods: b) non-normalization, c) median normalization, d) TIC normalization, e) root mean square (RMS) normalization, and f) labeled normalization. Labeled normalization generated the best fit $\left(R^{2}=0.985\right)$. The other normalization methods were better suited to non-linear curves, such as logarithmic regression. The correlation coefficients obtained for logarithmic regression were non-normalization, 0.902; median normalization, 0.926; TIC normalization, 0.868; RMS normalization, 0.937; and labeled normalization, 0.899. Reprinted from Kallback, P.; Shariatgorji, M.; Nilsson, A.; Andren, P., Novel mass spectrometry imaging software assisting labeled normalization and quantitation of drugs and neuropeptides directly in tissue sections. Journal of Proteomics 2012, 75, 4941-4951 (Ref [49]). Copyright (2012) with permission from Elsevier. 
A
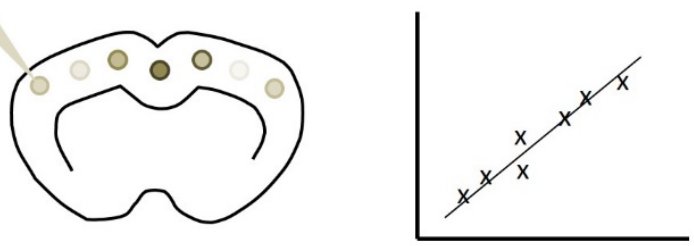

B

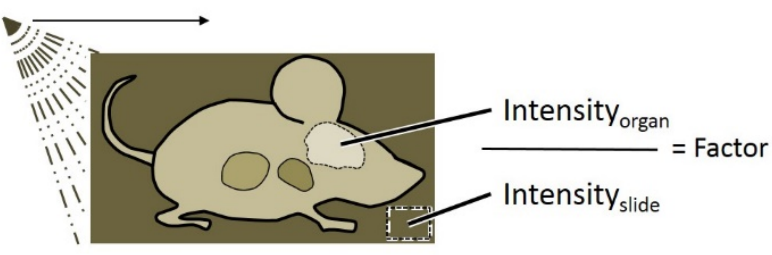

C
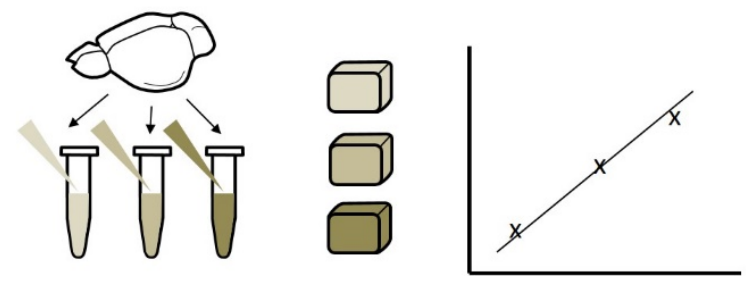

Figure 7. Schematic illustration of three strategies for offline Q-MSI using standards. A) Standard is spotted onto a tissue section at several concentrations, and the acquired intensities are used to generate an external calibration curve. B) Standard is sprayed uniformly onto a tissue section and glass slide, and the acquired intensities in one organ are normalized to the intensity of the standard on the glass slide to determine a tissue-specific response factor. C) Tissue is homogenized, and standard is added to the homogenate at several concentrations. The homogenate is solidified and sectioned, and the acquired intensities are used to generate an external calibration curve.

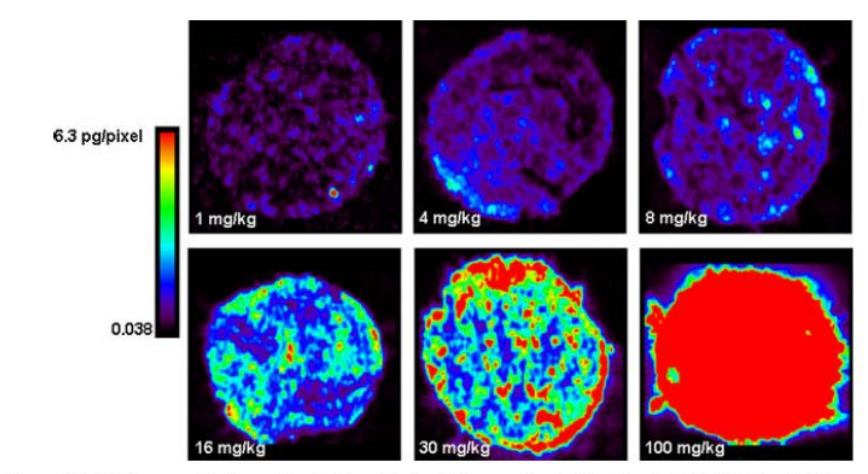

Figure 5. MSI images of olanzapine in liver obtained from animals dosed at 1, 4, 8, 16, 30 and $100 \mathrm{mg} / \mathrm{kg}$.

Figure 8. MSI images of olanzapine in liver obtained from animals dosed at 1, 4, 8, 16, 30, and 100 $\mathrm{mg} / \mathrm{kg}$. Used with permission from Koeniger, S. L.; Talaty, L.; Ready, D.; Voorbach, M.; Seifert, T.; Cepa, S.; Fagerland, J. A.; Bouska, J.; Buck, W.; Johnson, R. W.; Spanton, S., A quantitation method for mass spectrometry imaging. Rapid Communication in Mass Spectrometry, 2011, 25, 503-510 (Ref [74]). Copyright (2011) Wiley. 


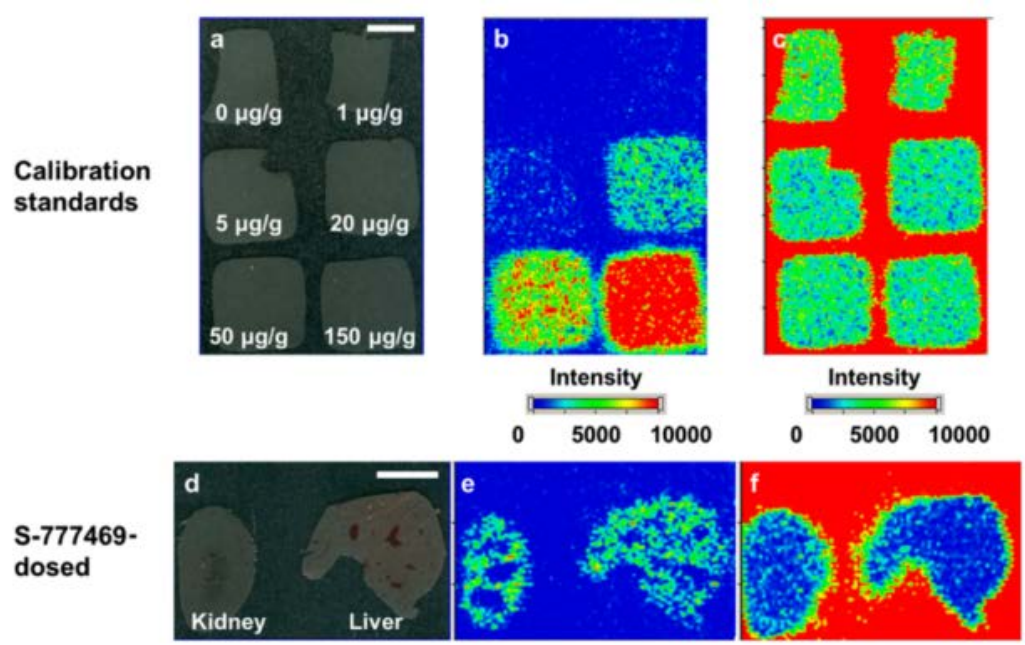

Figure 9. Representative optical images ( $a$ and d) and ion images of $m / z 415 \rightarrow 369$ ( $b$ and e) and $m / z$ $425 \rightarrow 379$ ( $c$ and $f$ ) on the sections of the calibration standards $(a-c)$ or the kidney and liver ( $d-f$ ). Bar = $55 \mathrm{~mm}$. Reproduced with permission from Takai, N.; Tanaka, Y.; Saji, H., Quantification of Small Molecule Drugs in Biological Tissue Sections by Imaging Mass Spectrometry Using Surrogate TissueBased Calibration Standards. Mass Spectrometry (Tokyo), 2014, 3(1): A0025 (Ref [68]).

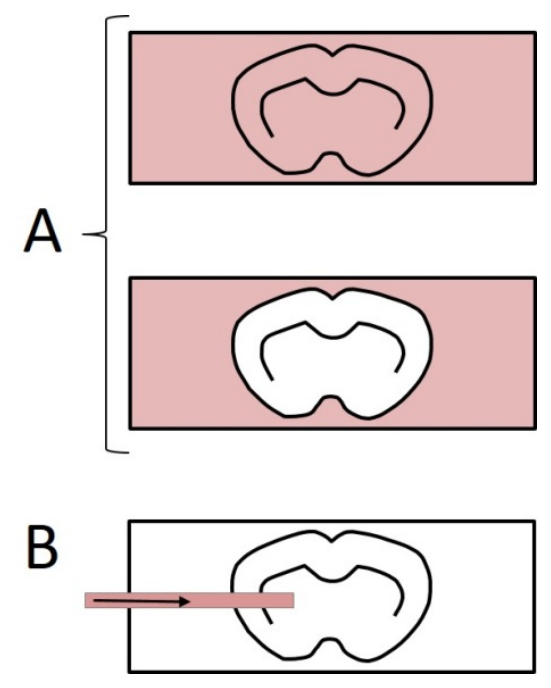

Figure 10. Schematics of the on-line quantification strategies. A) Uniform deposition of an internal standard on top of or under the tissue section. B) Internal standard incorporated into the solvent used for liquid extraction. 


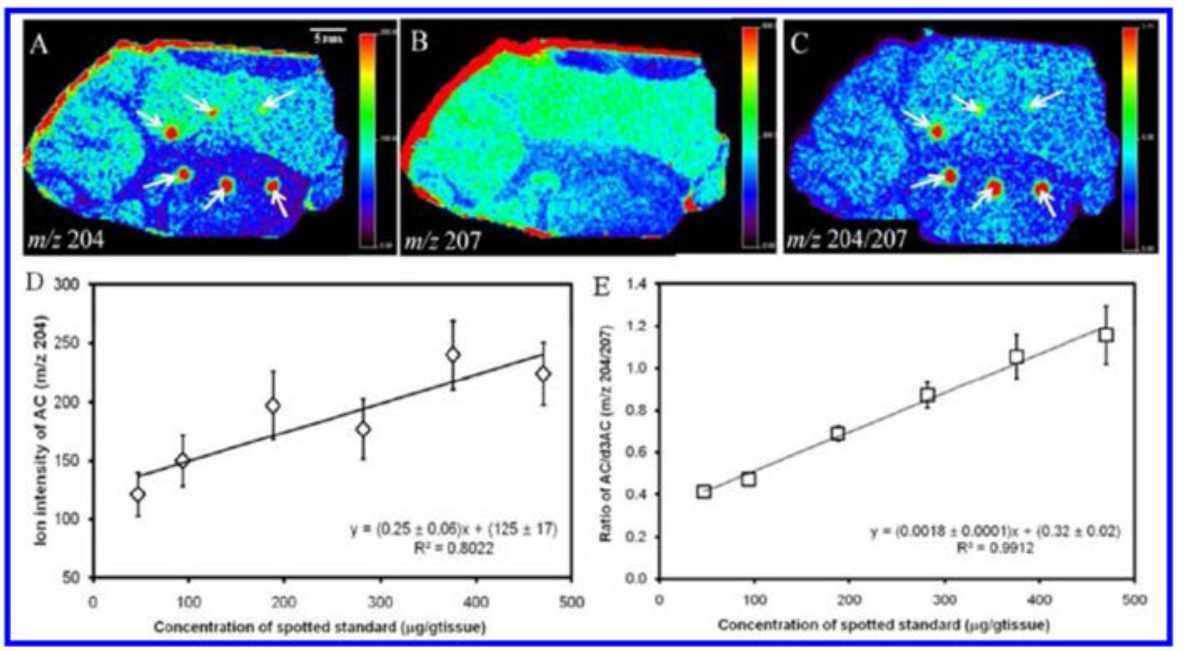

Figure 11. MS image of piglet brain tissue for the detection of endogenous acetylcarnitine. (A) Image of the $[\mathrm{M}+\mathrm{H}]+$ ion corresponding to acetylcarnitine $(\mathrm{m} / \mathrm{z} 204)$ shows a clear differentiation between the brain's white and gray matter. (B) Applied acetylcarnitine-d3 shows a similar pattern, implying there is either decreased extraction or ionization from the two tissue types. (C) Ratio image of acetylcarnitine/acetylcarnitine- $d 3$ results in a better representation of the distribution of acetylcarnitine within the brain sample while improving the calibration curve's linearity. Calibration curves plotted with (D) the ion intensity of $m / z 204$ versus (E) the ratio of $m / z \quad 204 / 207$. The appearance of saturated calibration spots is graphical due to scaling to show the endogenous levels of acetylcarnitine. Reprinted with permission from Pirman, D. A.; Kiss, A.; Heeren, R. M. A.; Yost, R. A., Identifying Tissue-Specific Signal Variation in MALDI Mass Spectrometric Imaging by Use of an Internal Standard. Analytical Chemistry 2013, 85, 1090-1096 (Ref [53]). Copyright (2013) American Chemical Society.

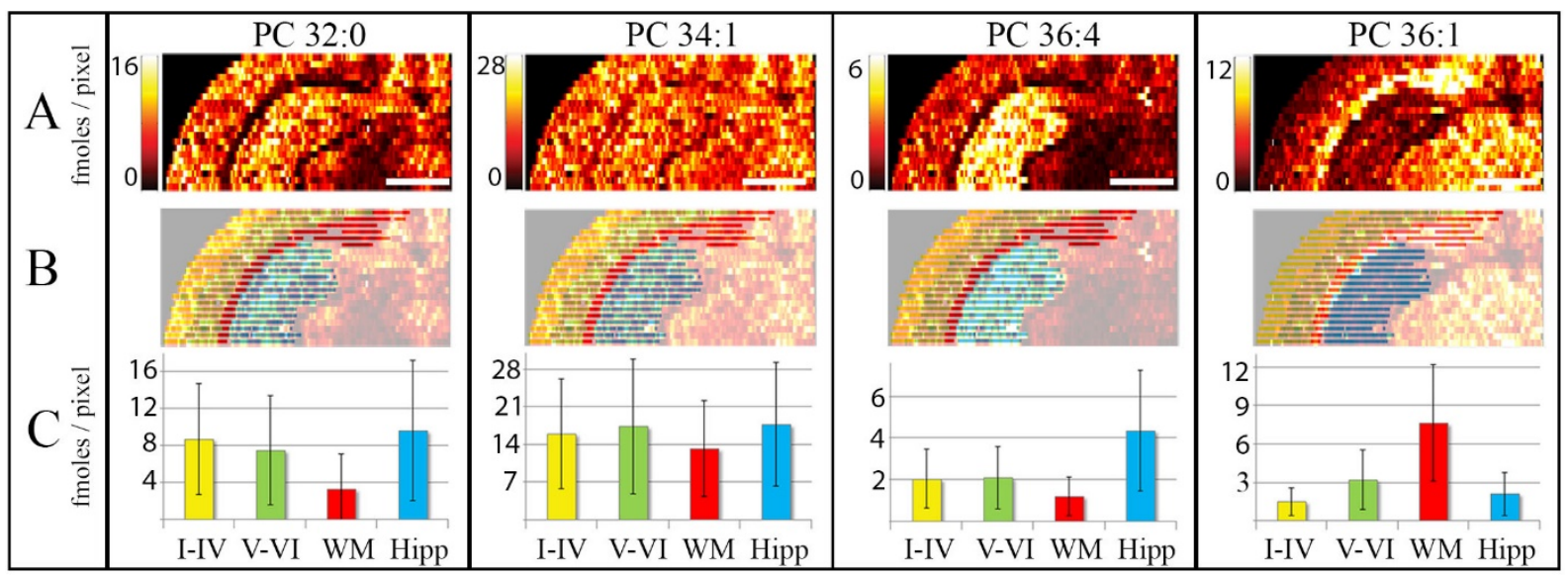

Figure 12. Quantitative ion images acquired using nano-DESI MSI including internal standards in the solvent. (A) Four quantitative ion images of endogenous PC. From left to right: PC 32:0, PC 34:1, PC 36:4, and $P C$ 36:1 are displayed as $[\mathrm{M}+\mathrm{K}]^{+}$signals normalized to $[\mathrm{PC} 43: 6+\mathrm{K}]^{+}$. The values in the intensity scale bar show fmol/pixel and vary between ion images. Scale bar shows $2 \mathrm{~mm}$. (B) Regions of interest are color coded as: yellow, neocortical layers I-IV; green, neocortical layers V-VI; red, white matter (WM); and blue, hippocampus (Hipp). (C) Graphs showing the average amount of the 
corresponding PC in each region of the brain in fmoles per pixel (color coded as described in B). Error bars show standard deviations of individual pixels in the respective region. Number of pixels within each region: I-IV, $\mathrm{n}=258 ; \mathrm{V}-\mathrm{VI}, \mathrm{n}=356 ; \mathrm{WM}, \mathrm{n}=249$; and Hipp, $\mathrm{n}=477$. Reprinted with permission from Lanekoff, I.; Thomas, M.; Laskin, J., Shotgun Approach for Quantitative Imaging of Phospholipids Using Nanospray Desorption Electrospray Ionization Mass Spectrometry. Analytical Chemistry 2014, 86, 1872-1880 (Ref [43]). Copyright (2014) American Chemical Society.
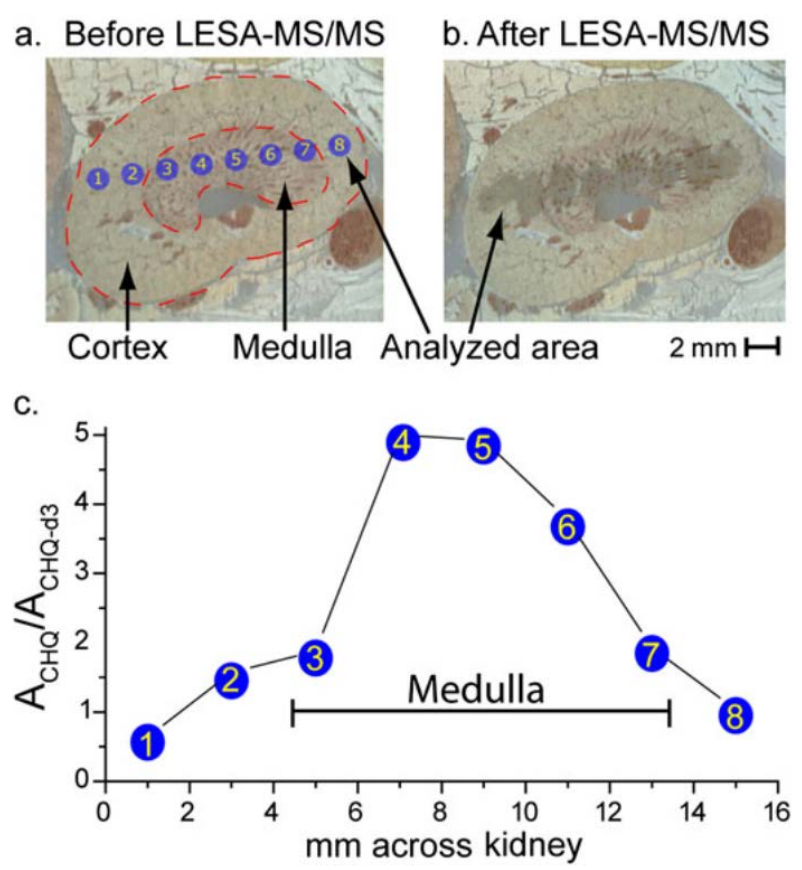

Figure 13. Spatial distribution of chloroquine (CHQ) in the kidney from a CHQ-dosed animal tissue section (10 mg/kg; $2 \mathrm{~h}$ ). (a) Optical image of the kidney tissue section prior to LESA-MS/MS analysis. The numbered blue circles were created using LESA; clarity software $(1 \mathrm{~mm}$ diameter with $2 \mathrm{~mm}$ center-to-center spacing) and selected LESA-MS/MS analysis points. (b) Optical image of kidney section after LESA-MS/MS analysis illustrating the actual areas analyzed across the cortex and medulla (outlined by red dashes in (a)). Each analysis spot was approximately 1.5-2 mm in diameter. (c) Integrated selected reaction monitoring signals of $\mathrm{CHQ}$ normalized to the internal standard, $\mathrm{CHQ}$ $\mathrm{d} 4$, are plotted versus distance in $\mathrm{mm}$ across the kidney section. The numbered blue circles correlate with the numbered circles in (a) and indicate the intensity versus spatial location in the cortex (circles 1, 2, and 8) and the medulla (circles 3-7). Used with permission from Parson, W. B.; Koeniger, S. L.; Johnson, R. W.; Erickson, J.; Tian, Y.; Stedman, C.; Schwartz, A.; Tarcsa, E.; Cole, R.; Van Berkel, G. J., Analysis of chloroquine and metabolites directly from whole-body animal tissue sections by liquid extraction surface analysis (LESA) and tandem mass spectrometry. Journal of Mass Spectrometry 2012, 47, 1420-1428 (Ref [52]). Wiley. 